\title{
Coexistence stability in a four-member hypercycle with error tail through center manifold analysis
}

\author{
Gerard Farré, ${ }^{1}$ Josep Sardanyés, ${ }^{2,3}$ Antoni Guillamon, ${ }^{4,3}$ and Ernest Fontich ${ }^{5,3}$ \\ ${ }^{1}$ Department of Mathematics (KTH, Royal Institute of Technology), SE-100 44 Stockholm \\ ${ }^{2}$ Centre de Recerca Matemàtica, Campus de Bellaterra, Edifici C, 08193 Bellaterra, Barcelona \\ ${ }^{3}$ Barcelona Graduate School of Mathematics BGSMath \\ ${ }^{4}$ Departament de Matemàtiques (Universitat Politècnica de Catalunya), Av. Gregorio Marañon 44-50, 08028 Barcelona \\ ${ }^{5}$ Departament de Matemàtiques i Informàtica (Universitat de Barcelona), \\ Gran Via de les Corts Catalanes 585, 08007 Barcelona
}

(Dated: September 27, 2017)

\begin{abstract}
Establishing the conditions allowing for the stable coexistence in hypercycles has been a subject of intensive research in the past decades. Deterministic, time-continuous models have indicated that, under appropriate parameter values, hypercycles are bistable systems, having two asymptotically stable attractors governing coexistence and extinction of all hypercycle members. The nature of the coexistence attractor is largely determined by the size of the hypercycle. For instance, for 2-member hypercycles the coexistence attractor is a stable node. For larger dimensions more complex dynamics appear. Numerical results on so-called elementary hypercycles with $n=3$ and $n=4$ species revealed, respectively, coexistence via strongly- and weakly-damped oscillations. Stability conditions for these cases have been provided by linear stability and Lyapunov functions. Typically, linear stability analysis of 4-member hypercycles indicates two purely imaginary eigenvalues and two negative real eigenvalues. For this case, stability can not be fully characterized by linearizing near the fixed point. In this letter we determine the stability of a non-elementary 4-member hypercycle which considers exponential and hyperbolic replication terms under mutation giving place to an error tail. Since Lyapunov functions are not available for this case, we use the center manifold theory to rigorously show that the system has a stable coexistence fixed point. Our results also show that this fixed point can not undergo a Hopf bifurcation, as supported by numerical simulations previously reported.
\end{abstract}

Keywords: Center manifold theory; Cooperation; Hypercycles; Nonlinear dynamics; Origins of Life.

\section{Introduction}

The stability of hypercycles is a very important subject of research within the-origin-of-life framework. Hypercycles are catalytic sets of macromolecules that can catalyze their own replication or the replication of other species of the network, which usually has cyclic architecture [? ]. Catalytic sets have been a subject of study in different fields. For instance, hypercycles have been largely investigated in the framework of prebiotic evolution [? ? ? ? ], suggested of being responsible for one of the major transitions in evolution: the transition from quasispecies to hypercycles as a possible way to surpass the information crisis at early stages of biological evolution [? ? ? ]. Hypercycle equations have been also used to study the dynamics of other complex systems. For instance, ecological dynamical systems [? ? ? ? ].

The conditions allowing the asymptotic persistence of hypercycles have been investigated during the past decades. The nature of the coexistence attractor for small hypercycles is well known. For instance, when $n=2$, the coexistence attractor is a stable node [? ? ? ? ] since linearization near these equilibria revealed two negative eigenvalues. For larger hypercycles the nature of the coexistence attractor slightly changes. Specifically, the stability for the so-called elementary hypercycle in [? ] has been determined by using Lyapunov functions. Together with these results, further numerical simulations have revealed that hypercycles with $n=3$ and $n=4$ have, respectively, a coexistence attractor which is achieved via strongly- and weakly-damped oscillations [? ? ]. For the $n=4$ dimension, linear stability analysis shows two imaginary eigenvalues with zero real part and two negative eigen- values. This particular condition does not allow to characterize the stability of this putative attractor. As mentioned, in the elementary hypercycle studied in Ref. [? ] the stability for $n=4$ was determined using a Lyapunov function. For the system explored in this letter, which considers the hypercycle with the error tail, it is not clear at all how to find a Lyapunov function. However, a rigorous stability analysis can be performed using the center manifold theory.

In this letter we perform this analysis showing that the dynamics restricted to the center manifold corresponding to the two imaginary eigenvalues is a stable (degenerate) focus of order two, which implies that this equilibrium point is certainly an attractor. Our study also shows that the stable fixed point can not undergo a Hopf bifurcation, thus no periodic orbits can born from this equilibrium point.

\section{Mathematical model}

The hypercycle model we analyze describes the time evolution of the relative concentration of $n$ molecular species with the so-called error tail [? ? ]. The hypercycle was conceived as a set of replicators with catalytic couplings replicating at extremely high mutation rates. Hence, more realistic hypercycle models need to consider the mutant replicators, which can be grouped as a single variable by defining the so-called errortail [? ? ], comprised for all those sequences with nucleotide changes arising within the population evolutionary dynamics. If we denote by $\left\{I_{1}, \ldots, I_{n}\right\}$ the hypercycle species and $I_{e}$ the species forming the error tail, the differential equations describing their concentrations $x=\left(x_{1}, \ldots, x_{n}\right)$ and $x_{e}$, which 
are real-valued variables, are

$$
\dot{x}_{j}=x_{j}\left(A_{j} Q+K_{j} x_{j-1} Q-\hat{\Phi}\left(x, x_{e}\right)\right),
$$

for $j=1, \ldots, n$, and

$$
\dot{x}_{e}=x_{e}\left(A_{e}-\hat{\Phi}\left(x, x_{e}\right)\right)+(1-Q) \sum_{j=1}^{n} x_{j}\left(A_{j}+K_{j} x_{j-1}\right),
$$

where $x_{0} \equiv x_{n}, K_{j}, A_{j}>0,1 \leq j \leq n, A_{e}>0, Q \in(0,1)$ and

$$
\hat{\Phi}\left(x, x_{e}\right)=\sum_{j=1}^{n} x_{j}\left(A_{j}+K_{j} x_{j-1}\right)+A_{e} x_{e} .
$$

System (1) describes the dynamics of the hypercycle of dimension $n$ with both Malthusian and heterocatalytic (nonlinear) reproduction together with mutation. Both sets of constants $A_{j}$ and $K_{j}$, for $j=1, \ldots, n$, denote the Malthusian and heterocatalytic replication rates, respectively. The parameter $Q$ is the replication quality factor or copying fidelity. Equation (2) corresponds to the dynamics of the error tail. The solutions of the full system (1)-(2) live in the $(n+1)$-dimensional real space $\mathbb{R}^{n+1}$. Finally, $\hat{\Phi}\left(x, x_{e}\right)$ is the dilution flow that keeps the total population constant; it also introduces competition between all the replicators forming the hypercycle and the error tail.

The hyperplane $H=\left\{\left(x, x_{e}\right) \in \mathbb{R}^{n+1} \mid \sum_{j=1}^{n} x_{j}+x_{e}=1\right\}$ is invariant by the flow. Accordingly, we will restrict our study to the solutions in $H$. Moreover, we can forget about the error tail and omit the term $x_{e}$ because it is given by $x_{e}=1-$ $\sum_{j=1}^{n} x_{j}$. Thus we are let to consider the differential equation

$$
\dot{x}_{j}=f_{j}(x)=x_{j}\left(A_{j} Q+K_{j} x_{j-1} Q-\Phi(x)\right),
$$

$1 \leq j \leq n$, where $\Phi(x)=\sum_{j=1}^{n} x_{j}\left(A_{j}+K_{j} x_{j-1}\right)+A_{e}(1-$ $\left.\sum_{j=1}^{n} x_{j}\right)$ and $x=\left(x_{1}, \ldots, x_{n}\right)$.

We will restrict to the biological meaningful region, namely the set

$$
S=\left\{\left(x_{1}, \ldots, x_{n}\right) \mid 0 \leq x_{j} \leq 1, \sum_{j=1}^{n} x_{j} \leq 1\right\},
$$

which is positively invariant by the evolution of the hypercycle. That is, the flow will never come out from $S$ and it will exist for any positive time. Additionally, we will assume that $K_{j}=1$ to simplify the model.

\section{The symmetric hypercycle}

We will take $A_{j}=A_{e}=a, \forall j \in\{1, \ldots, n\}$, for some $a>$ 0 . We refer to this case as the symmetric hypercycle, which assumes that all hypercycle elements are selectively neutral. The following proposition gives the equilibrium points in any dimension. For the sake of completeness, we include the proof in Appendix 1.
Proposition 1 For the values of $(a, Q)$ such that $Q^{2} /(1-$ $Q) \geq 4 n a$, equation (3) has three fixed points in $S: x_{0}=$ $(0, \ldots, 0)=\overrightarrow{0}$, and $x_{+}^{*}, x_{-}^{*}$, whose coordinates are

$$
x_{ \pm, j}^{*}=\frac{Q \pm \sqrt{Q^{2}-4 n a(1-Q)}}{2 n},
$$

for all $j \in\{1, \ldots, n\}$. When $Q^{2} /(1-Q)<4$ na, the only fixed point of equation (3) in $S$ is the origin.

The coordinates of the non trivial equilibrium points are actually the two solutions of $n x^{2}-Q x+a(1-Q)=0$. Notice that for $Q=Q_{S S}:=2(\sqrt{n a(1+n a)}-n a)$ a saddle-node bifurcation occurs and the two equilibria $x_{+}^{*}$ and $x_{-}^{*}$ appear for $Q>Q_{S S}$.

\section{Stability of the equilibrium points}

In this section we will classify the character of the fixed points. We first note that the right-hand side of (3) for the symmetric case can be written as

$$
f_{j}(x)=x_{j} F_{j}(x), \quad j \in\{1, \ldots, n\},
$$

where $F_{j}(x)=a(Q-1)+Q x_{j-1}-\sum_{k=1}^{n} x_{k} x_{k-1}$.

Since

$$
\frac{\partial f_{j}}{\partial x_{k}}(x)=\delta_{j k} F_{j}(x)+x_{j} \frac{\partial F_{j}}{\partial x_{k}}(x),
$$

where $\delta_{j k}$ is the Kronecker delta function and

$$
\frac{\partial F_{j}}{\partial x_{k}}(x)= \begin{cases}Q-x_{k+1}-x_{k-1}, & k=j-1, \\ -x_{k+1}-x_{k-1}, & k \neq j-1,\end{cases}
$$

it turns out that the components of the Jacobian of $f$ are

$$
\frac{\partial f_{j}}{\partial x_{k}}(x)= \begin{cases}x_{j}\left(Q-x_{k+1}-x_{k-1}\right), & k=j-1, \\ x_{j}\left(-x_{k+1}-x_{k-1}\right), & k \notin\{j-1, j\}, \\ F_{j}(x)+x_{j}\left(-x_{j+1}-x_{j-1}\right), & k=j .\end{cases}
$$

Substitution of $x_{j}=0$, for all $j=1, \ldots, n$, into (5) leads to $\mathrm{D} f(\overrightarrow{0})=\operatorname{diag}(a(Q-1), \ldots, a(Q-1))$. Therefore the eigenvalues of $\mathrm{D} f(\overrightarrow{0})$ are all equal to $a(Q-1)<0$ so $x^{*}=$ $\overrightarrow{0}$ is an attracting fixed point independently of the parameter values $(a, Q), a>0, Q \in(0,1)$. In the same way we will analyze the stability of the fixed points $x_{+}^{*}$ and $x_{-}^{*}$ given in Proposition 1. Using again equation (5), together with the fact that all the components are equal, we obtain the following expression for the entries of the Jacobian matrix

$$
\frac{\partial f_{j}}{\partial x_{k}}\left(x_{ \pm}^{*}\right)= \begin{cases}x_{ \pm, 1}^{*}\left(Q-2 x_{ \pm, 1}^{*}\right), & k=j-1, \\ -2\left(x_{ \pm, 1}^{*}\right)^{2}, & k \notin\{j-1, j\}, \\ F_{j}\left(x_{ \pm, 1}^{*}\right)-2\left(x_{ \pm, 1}^{*}\right)^{2}, & k=j .\end{cases}
$$

From the proof of Proposition 1, we know that

$$
a(Q-1)+Q x_{ \pm, 1}^{*}-n\left(x_{ \pm, 1}^{*}\right)^{2}=0 .
$$




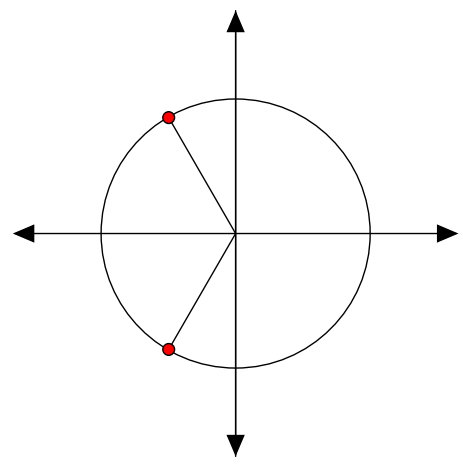

(a) $n=3$

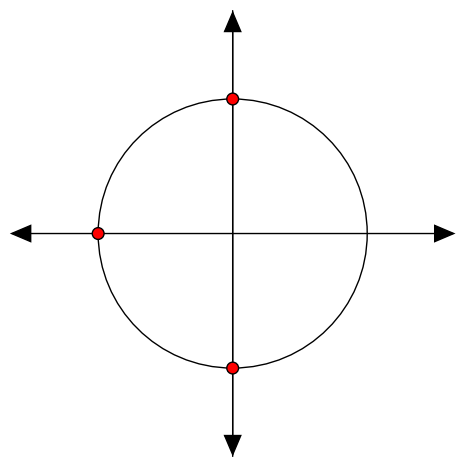

(b) $n=4$

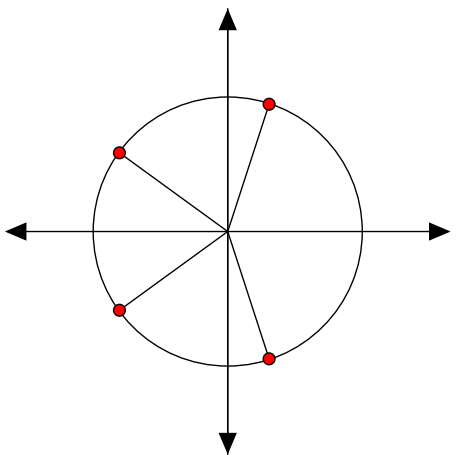

(c) $n=5$

Figure 1: Eigenvalues $\lambda_{j}^{+}$for $0<j<n$ on the circle of radius $k=Q x_{+, 1}^{*}$ in the complex space $\mathbb{C}$. The case $n=4$, analyzed in this letter, displays two eigenvalues with negative real part and a couple of complex eigenvalues with zero real part $\left(\lambda_{0}^{+}\right.$is not displayed).

Therefore if we denote $b_{ \pm}:=-2\left(x_{ \pm, 1}^{*}\right)^{2}$ and $d_{ \pm}:=$ $x_{ \pm, 1}^{*}\left(Q-2 x_{ \pm, 1}^{*}\right)$,

$$
\mathrm{D} f\left(x_{ \pm}^{*}\right)=\left(\begin{array}{ccccc}
b_{ \pm} & b_{ \pm} & \ldots & b_{ \pm} & d_{ \pm} \\
d_{ \pm} & \ddots & \ddots & \ddots & b_{ \pm} \\
b_{ \pm} & \ddots & \ddots & \ddots & \vdots \\
\vdots & \ddots & \ddots & \ddots & b_{ \pm} \\
b_{ \pm} & \ldots & b_{ \pm} & d_{ \pm} & b_{ \pm}
\end{array}\right)
$$

which is a circulant matrix. There are explicit formulas to get the eigenvalues and the eigenvectors for circulant matrices, see e.g., [? ]. In our case, these formulas give

$$
\begin{aligned}
\lambda_{j}^{ \pm}= & \sum_{k=0}^{n-2}-2\left(x_{ \pm, 1}^{*}\right)^{2} \exp (2 \pi i j k / n) \\
& +x_{ \pm, 1}^{*}\left(Q-2 x_{ \pm, 1}^{*}\right) \exp (2 \pi i j(n-1) / n),
\end{aligned}
$$

where $i^{2}=-1$ and $j \in\{0, \ldots, n-1\}$. If $j \neq 0$, using that the sum of the geometric progression $\sum_{k=0}^{n-1} \exp (2 \pi i j k / n)$ is zero, we can simplify (7) to obtain:

$$
\lambda_{j}^{ \pm}=x_{ \pm, 1}^{*} Q \exp (2 \pi i j(n-1) / n), \quad j \in\{1, \ldots, n-1\} .
$$

For $j=0$ we get:

$$
\begin{aligned}
\lambda_{0}^{ \pm} & =x_{ \pm, 1}^{*}\left(Q-2 x_{ \pm, 1}^{*}\right)-2(n-1)\left(x_{ \pm, 1}^{*}\right)^{2} \\
& =Q x_{ \pm, 1}^{*}-2 n\left(x_{ \pm, 1}^{*}\right)^{2}=-Q x_{ \pm, 1}^{*}+2 a(1-Q),
\end{aligned}
$$

using (6) again.

Observe that for $x_{-}^{*}$ we have that $\lambda_{0}^{-}>0$, and so $x_{-}^{*}$ is unstable independently of the character of other eigenvalues. On the other hand, since $\lambda_{0}^{+}=x_{+, 1}^{*}\left(Q-2 n x_{+, 1}^{*}\right)$ and $x_{+, 1}^{*}>Q /(2 n)>0$, then $\lambda_{0}^{+}<0$. Thus, to determine the stability character of $x_{+}^{*}$ we need to study the sign of the real part of $\lambda_{j}^{+}$for $0<j \leq n$. As it is illustrated in Figure 1, we have that for $n \leq 3$ all these eigenvalues have negative real part and for $n \geq 5$ we will always have eigenvalues with positive real part. Therefore for $n \leq 3$ the equilibrium point $x_{+}^{*}$ is an attractor and for $n \geq 5$ it is unstable. It remains the discussion in the case $n=4$, for which $x_{+}^{*}$ is non hyperbolic.

\section{Stability of $x_{+}^{*}$ when $n=4$}

In order to simplify the notation, we introduce the parameters

$$
\kappa:=Q x_{+, 1}^{*} \text { and } c:=\kappa-2 a(1-Q) .
$$

Notice that both $\kappa$ and $c$ are positive. Then, for $n=4$, the eigenvalues of $\mathrm{D} f\left(x_{ \pm}^{*}\right)$ read as

$$
\begin{array}{ll}
\lambda_{0}^{+}=-c, & \lambda_{1}^{+}=\kappa e^{2 \pi i \frac{3}{4}}=-i \kappa, \\
\lambda_{2}^{+}=\kappa e^{2 \pi i \frac{3}{2}}=-\kappa, & \lambda_{3}^{+}=\kappa e^{2 \pi i \frac{9}{4}}=i \kappa,
\end{array}
$$

and their corresponding eigenvectors are:

$$
\begin{array}{ll}
v_{0}=(1,1,1,1)^{\top}, & v_{1}=(1, i,-1,-i)^{\top}, \\
v_{2}=(1,-1,1,-1)^{\top}, & v_{3}=(1,-i,-1, i)^{\top} .
\end{array}
$$

The invariant subspace generated by $v_{1}$ and $v_{3}$ (thought as a vector space over the complex space $\mathbb{C}$ ) is also generated by $w_{1}:=\frac{1}{2}\left(v_{1}+v_{3}\right), w_{2}:=\frac{i}{2}\left(v_{3}-v_{1}\right)$ and moreover,

$$
\begin{aligned}
& \operatorname{Df}\left(x_{ \pm}^{*}\right) w_{1}=-\frac{i}{2} \kappa v_{1}+\frac{i}{2} \kappa v_{3}=\kappa w_{2}, \\
& \operatorname{Df}\left(x_{ \pm}^{*}\right) w_{2}=-\frac{1}{2} \kappa v_{1}-\frac{1}{2} \kappa v_{3}=-\kappa w_{1} .
\end{aligned}
$$

This linear study is not sufficient to draw conclusions about the stability of the equilibrium $x_{+, 1}^{*}$. At this point, we know that two eigenvalues are negative and the other two have zero real part. Moreover, in this situation, the point $x_{+}^{*}$ has a center manifold which is tangent to the linear space generated by $v_{1}$ and $v_{3}$. If we are able to prove that the equilibrium point of the system restricted to the center manifold is asymptotically stable, then by Theorem 2 in [?, Ch. 1] we will prove that 
the equilibrium of the four dimensional system (3) is asymptotically stable. Let us then compute the center manifold and study the restriction of (3) on it.

To reduce the vector field to the central manifold it is convenient to perform a translation of the point $x_{+}^{*}$, say $z=x-x_{+}^{*}$ to the origin followed by a linear change $z=C y$, where $C$ is the matrix whose columns are the vectors $w_{1}, w_{2}, v_{2}$ and $v_{0}$, that is,

$$
C=\left(\begin{array}{cccc}
1 & 0 & 1 & 1 \\
0 & 1 & -1 & 1 \\
-1 & 0 & 1 & 1 \\
0 & -1 & -1 & 1
\end{array}\right)
$$

In the new set of variables $y=\left(y_{1}, y_{2}, y_{3}, y_{4}\right)$, the vector field is expressed as

$$
\dot{y}=C^{-1} f\left(C y+x_{+}^{*}\right)=: G(y),
$$

which is topologically conjugate to (3) since translations and invertible linear maps are diffeomorphisms.

Performing the whole calculation and using the fact that $x_{+, 1}^{*}$ is a solution of (6), we can express $G(y)=G[1]+G[2]+G[3]$, where the index stands for the degree of the polynomial in $y$ and

$$
\begin{aligned}
& G[1]=\left(\begin{array}{c}
-\kappa y_{2} \\
\kappa y_{1} \\
-\kappa y_{3} \\
-c y_{4}
\end{array}\right) \\
& G[2]=\left(\begin{array}{c}
Q\left(y_{1}\left(y_{4}-y_{3}\right)-y_{2}\left(y_{3}+y_{4}\right)\right)-8 x_{+, 1}^{*} y_{1} y_{4} \\
Q\left(y_{1}\left(y_{4}-y_{3}\right)+y_{2}\left(y_{3}+y_{4}\right)\right)-8 x_{+, 1}^{*} y_{2} y_{4} \\
-\left(Q y_{1} y_{2}+8 x_{+, 1}^{*} y_{3} y_{4}\right) \\
\left(-Q+4 x_{+}^{*}\right) y_{3}^{2}+\left(Q-12 x_{+, 1}^{*}\right) y_{4}^{2}
\end{array}\right) \\
& G[3]=4\left(y_{3}^{2}-y_{4}^{2}\right)\left(\begin{array}{l}
y_{1} \\
y_{2} \\
y_{3} \\
y_{4}
\end{array}\right)
\end{aligned}
$$

Notice that now the linear part of the system corresponds to the expected one. The above expressions were obtained using the computer algebra software Maple [? ] (code available at http://bit.ly/2tgxlus), but we also include the full set of computations in Appendix 2 for the sake of completeness.

\section{Reduction to the center manifold}

The resulting system (10) is polynomial of degree 3 and therefore a $\mathcal{C}^{\infty}$ function (i.e., is a function that is differentiable for all degrees of differentiation). Thus by the center manifold theorem [?] we know that $\overrightarrow{0}$ has a center manifold $W^{c}=\operatorname{graph}(h)$ tangent to the vector space generated by the eigenvectors corresponding to the eigenvalues with zero real part, that is, the plane generated by $y_{1}$ and $y_{2}$. The center manifold is locally parameterized by a $\mathcal{C}^{r}$ function $h$ with $r$ as large as we want. We rewrite (10) in the form

$$
\left\{\begin{array}{l}
\dot{\xi}=A \xi+u(\xi, \eta), \\
\dot{\eta}=B \eta+v(\xi, \eta)
\end{array}\right.
$$

with $\xi=\left(y_{1}, y_{2}\right), \eta=\left(y_{3}, y_{4}\right)$,

$$
A=\left(\begin{array}{cc}
0 & -\kappa \\
\kappa & 0
\end{array}\right) \quad \text { and } B=\left(\begin{array}{cc}
-\kappa & 0 \\
0 & -c
\end{array}\right) \text {, }
$$

and $u(\xi, \eta)$ and $v(\xi, \eta)$ represent the nonlinear terms of the system.

In this notation, $W^{c}$ is represented by $\eta=h(\xi)$. Then, the condition of $\operatorname{graph}(h)$ being invariant by (12) is $\dot{\eta}=D h(\xi) \dot{\xi}$, that is,

$$
B \eta+v(\xi, \eta)=D h(\xi)(A \xi+u(\xi, \eta)) .
$$

Substituting $\eta=h(\xi)$ into equation (13) gives

$$
B h(\xi)+v(\xi, h(\xi))=D h(\xi)(A \xi+u(\xi, h(\xi))) .
$$

From now on, we rename variables $y_{1}$ and $y_{2}$ by $x$ and $y$, respectively, so that $\xi=(x, y)$. For our purposes it is enough to know the quadratic terms of the function $h$; accordingly, we write

$$
h(x, y)=\left(\begin{array}{c}
a_{20} x^{2}+a_{11} x y+a_{02} y^{2} \\
b_{20} x^{2}+b_{11} x y+b_{02} y^{2}
\end{array}\right)+\mathcal{O}(3),
$$

where $\mathcal{O}(3)$ denotes all the terms of order 3 or greater. Substituting (15) into (14), we have

$$
\begin{gathered}
\left(\begin{array}{c}
-\kappa a_{20} x^{2}-\kappa a_{02} y^{2}-\left(\kappa a_{11}+Q\right) x y \\
-c\left(b_{20} x^{2}+b_{02} y^{2}+b_{11} x y\right)
\end{array}\right) \\
=\kappa\left(\begin{array}{c}
a_{11} x^{2}-a_{11} y^{2}+2\left(a_{02}-a_{20}\right) x y \\
b_{11} x^{2}-b_{11} y^{2}+2\left(b_{02}-b_{20}\right) x y
\end{array}\right),
\end{gathered}
$$

from which we obtain

$$
\begin{gathered}
a_{11}=-\frac{Q}{5 \kappa}, \quad a_{20}=\frac{Q}{5 \kappa}, \quad a_{02}=-\frac{Q}{5 \kappa} \\
b_{11}=0, \quad b_{20}=0, \quad b_{02}=0 .
\end{gathered}
$$

Thus, our function $h$ will be

$$
h(x, y)=\left(\begin{array}{c}
\frac{Q}{5 \kappa}\left(x^{2}-x y-y^{2}\right) \\
0
\end{array}\right)+\mathcal{O}(3)
$$

Now, we can write the system restricted to the center manifold, $A\left(\begin{array}{l}x \\ y\end{array}\right)+u(x, y, h(x, y))$, which reads as

$$
\left\{\begin{array}{l}
\dot{x}=-\kappa y-\frac{Q^{2}}{5 \kappa}\left(x^{3}-2 x y^{2}-y^{3}\right)+\mathcal{O}(4) \\
\dot{y}=\kappa x-\frac{Q^{2}}{5 \kappa}\left(x^{3}-2 x^{2} y+y^{3}\right)+\mathcal{O}(4) .
\end{array}\right.
$$


A first attempt to determine the stability of $\overrightarrow{0}$ is to change to polar coordinates $(r, \theta)$. The derivative of $r$ is:

$$
\begin{aligned}
\dot{r}=r^{3} \frac{Q^{2}}{5 \kappa}\left[\sin ^{3} \theta(\cos \theta-\sin \theta)\right. & -\cos ^{3} \theta(\cos \theta+\sin \theta) \\
& \left.+4 \cos ^{2} \theta \sin ^{2} \theta\right] .
\end{aligned}
$$
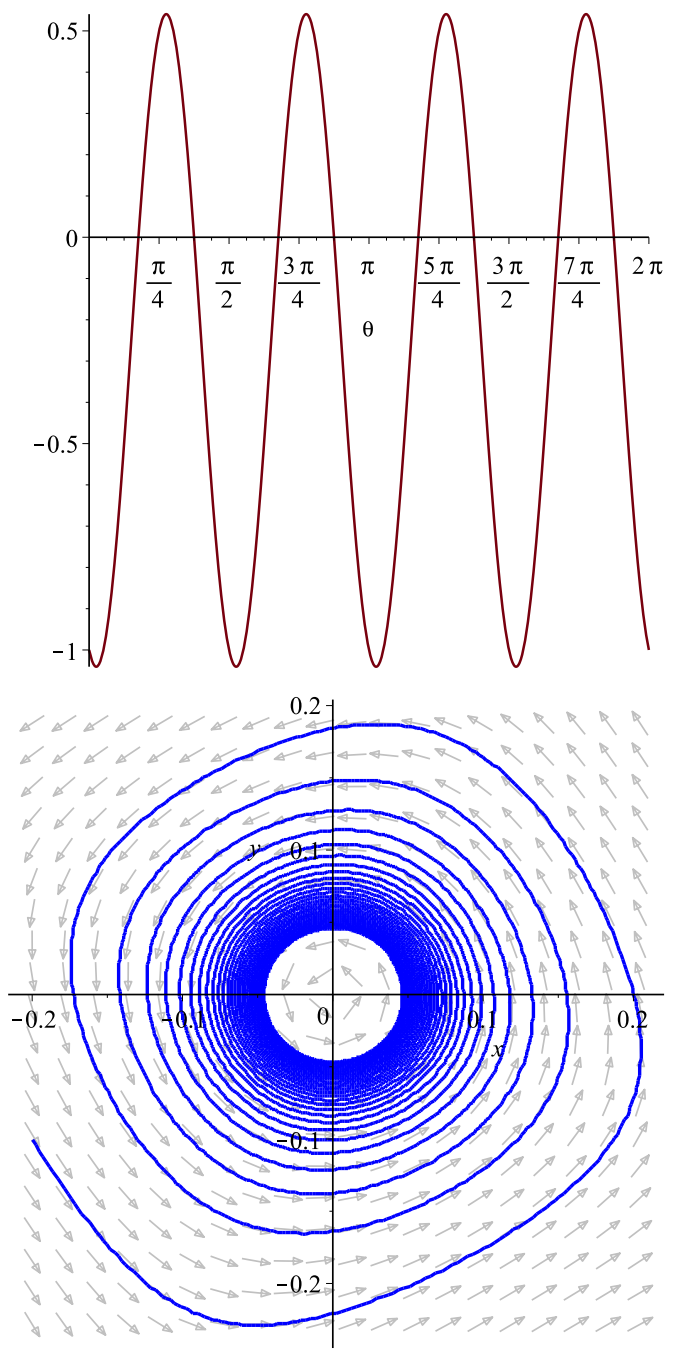

Figure 2: Radial derivative and phase portrait for system (16). (Upper) Plot of $\dot{r} / r^{3}$ with respect to $\theta$. (Lower) Flow for Eqs. (16) around $\overrightarrow{0}$ for $a=0.5, Q=0.95$. Grey arrows indicate the vector field.

We note that the sign of $\dot{r}$ depends on $\theta$, see Figure 2(upper), so it does not provide a conclusive information concerning stability. However, the flow of the vector field of system (16), see Figure 2(lower), shows evidence that the equilibrium point is stable.

In order to prove the stability, we will look for the normal form of the system at the origin, see [?, Ch. 3.3]. For this purpose it is convenient to write system (16) in complex variables, $z=$

$$
\begin{aligned}
& x+i y \text { and } \bar{z}=x-i y: \\
& \dot{z}=i \kappa z-(1+3 i) \frac{Q^{2}}{20 \kappa} z^{2} \bar{z}-(3+i) \frac{Q^{2}}{20 \kappa} \bar{z}^{3}+\mathcal{O}(4),
\end{aligned}
$$

and the equation for $\bar{z}$, which is the complex conjugate of equation (17). In these variables, the linear part is diagonal. An additional advantage of working in these variables is that we only need to transform one equation because the other remains its conjugate. Since the eigenvalues are $\pm i \kappa$, the resonant terms for the equation (17) are $z^{n+1} \bar{z}^{n}$, with $n \geq 1$. The explicit calculations give that the normal form of (17) is

$$
\dot{z}=i \kappa z-\frac{Q^{2}(1+3 i)}{20 \kappa} z^{2} \bar{z}+\mathcal{O}(5) .
$$

Alternatively, we can apply Theorem A in [? ] to system (17), see [?, Ch. IX.2] for a more classical reference, to conclude that $\overrightarrow{0}$ is an attracting equilibrium point. However, for this case we can proceed directly writing (18) in polar coordinates by means of the change $z=r e^{i \theta}$, which leads to

$$
\left\{\begin{array}{l}
\dot{r}=-\frac{Q^{2}}{20 \kappa} r^{3}+\mathcal{O}(5) \\
\dot{\theta}=\kappa-\frac{3 Q^{2}}{20 \kappa} r^{2}+\mathcal{O}(4)
\end{array}\right.
$$

Notice that near the origin $\dot{r}<0$ and $\dot{\theta}>0$, so it is clear that the origin is asymptotically stable, and so it is for system (16) (recall that the stability of the fixed points is preserved under locally topological conjugacies). Finally, as we pointed out at the end of Section III A, using Theorem 2 in [? , Ch. 1] we reach the conclusion that the fixed point $x_{+}^{*}$ in the original fourth dimensional system (3) is an attractor.

The fact that $\dot{r}<0$ for all values of the (considered) parameters prevents the birth of a periodic orbit from $\overrightarrow{0}$ (which corresponds to $x_{+}^{*}$ in the present variables), which implies that no Hopf bifurcation can take place.

\section{Discussion}

In this letter we have conducted a stability analysis using the center manifold theory for hypercycles with $n=4$ species and an error tail formed by mutant replicators. Previous works provided numerical evidences that the coexistence in hypercycles was governed by a stable fixed point [? ? ]. Numerical simulations indicated that such a fixed point can be achieved with different damped oscillatory modes (i.e., strongly-damped oscillations for $n=3$ and weakly-damped oscillations for $n=4$ ) [? ]. Moreover, for the so called elementary hypercycle in [?] Lyapunov functions are known for these fixed points when $n=3$ and $n=4$ so they are asymptotically stable. Despite these previous results, and for other types of hypercycles beyond the elementary ones, linear stability analysis around the coexistence attractor for $n=4$ gives two purely imaginary eigenvalues and two other negative eigenvalues. Since Lyapunov functions for these cases are not available, no direct conclusions about the stability of this fixed point can be attained. 
As mentioned above, we here explore the stability in the coexistence for a hypercycle with four species in a slightly different model from the one analyzed in [? ]. In particular, it introduces another layer of complexity by considering that replicators can synthesize neutral mutants, which ultimately act as parasites, since they do not reciprocate the catalytic support to the replicators forming the catalytic cycle. To determine the stability for this case we have performed a reduction to the center manifold, showing that this fixed point is locally asymptotically stable, in agreement with numerical results conducted for this type of hypercycle [? ].

Our approach also indicates that a Hopf bifurcation giving place to a periodic orbit underlying coexistence dynamics is not possible. In this sense, our approach reinforces the results suggesting that the minimal hypercycle size for which periodic orbits can exist is $n>4$ species [? ? ]. Our analytical results thus complement previous studies on the stability of hypercycles with the so-called error tail [? ? ]. Initially, hypercycle studies obviated the mutant replicators [? ], and focused on hyperycle dynamics without mutation processes. However, as previously mentioned, hypercycles, as prebiotic replicator systems, may be expected to replicate under large mutation rates. The stability for hypercycles with error-tails, specially for those systems with $n \geq 4$ replicators was performed mainly numerically [? ? ]. Here, we have provided rigorous arguments to assert that four-species hypercycles with error-tails are indeed stable under appropriate parameter values.

The analysis presented in this letter, albeit being purely theoretical, may have applications within the fields of synthetic biology and bioengineering. In this sense, experimental studies have characterized hypercycle dynamics in the bacteriophage $Q \beta$ [? ] and, more recently, in cooperating engineered yeast populations [?] and engineered synthetic bacteria with their parasites [? ]. Studies on the conditions allowing for the stability for cooperative systems thus become important and can allow to properly define those parameter regions where hypercycle systems may be stable and perform their functions, specially under the presence of mutant parasites.

\section{Acknowledgements}

This work has been partially funded by the Spanish grants MTM2013-41168-P, MTM2016-80117-P (MINECO/FEDER, UE) (EF) and MTM2015-71509-C2-2-R (MINECO/FEDER, UE) (AG), and the Catalan grants AGAUR 2014SGR-1145 (EF) and 2014SGR-504 (AG). JS has been partially funded by the CERCA Programme of the Generalitat de Catalunya. The research leading to these results has received funding from "la Caixa" Foundation.

\section{Appendix 1: Proof of Proposition 1}

Suppose we have an equilibrium point $x^{*} \in S$ (in particular all components are greater or equal than 0) such that $x_{j}^{*}=0$ for a fixed $0 \leq j<n$. Then since
$\dot{x}_{j+1}=x_{j+1}\left(a(Q-1)+Q x_{j}-\sum_{k=1}^{n} x_{k} x_{k-1}\right)$ we have either $x_{j+1}^{*}=0$ or

$0=a(Q-1)+Q x_{j}^{*}-\sum_{k=1}^{n} x_{k}^{*} x_{k-1}^{*}=a(Q-1)-\sum_{k=1}^{n} x_{k}^{*} x_{k-1}^{*}$.

This last case would imply

$$
\sum_{k=1}^{n} x_{k}^{*} x_{k-1}^{*}=a(Q-1)<0
$$

since $a>0$ and $Q<1$, leading to a contradiction because the left hand side should be positive or zero. Therefore $x_{j}^{*}=0$ implies $x_{j+1}^{*}=0$ for any $0 \leq j<n$ (recall we identify $\left.x_{0} \equiv x_{n}\right)$ and so if there is some $0 \leq j<n$ such that $x_{j}^{*}=0$ we must have $x^{*}=\overrightarrow{0}$. It is clear then that any fixed point $x^{*} \in S$ different from the origin must satisfy

$$
a(Q-1)+Q x_{j}^{*}-\sum_{k=1}^{n} x_{k}^{*} x_{k-1}^{*}=0, \quad \forall 1 \leq j \leq n,
$$

which can be rewritten as

$$
x_{j}^{*}=\frac{\sum_{k=1}^{n} x_{k}^{*} x_{k-1}^{*}-a(Q-1)}{Q}, \quad \forall 1 \leq j \leq n .
$$

From (20) we see that all components must be equal and so (19) becomes the quadratic equation

$$
a(Q-1)+Q x_{j}^{*}-n\left(x_{j}^{*}\right)^{2}=0,
$$

which has the same two solutions for all $j \in\{1, \ldots, n\}$ :

$$
x_{ \pm, 1}^{*}=\frac{Q \pm \sqrt{Q^{2}-4 n a(1-Q)}}{2 n} .
$$

It is easy to verify that if the discriminant in (21) is not negative (that is $Q^{2} /(1-Q) \geq 4 n a$ ), then such fixed points are contained in $S$ because $0<x_{ \pm, 1}^{*}<1 / n$ and so $\sum_{j=1}^{n} x_{ \pm, 1}^{*}=$ $n x_{ \pm, 1}^{*}<1$. If $Q^{2} /(1-Q)<4 n a$, then the components are complex and the only fixed point that remains in $S$ is the origin.

\section{Appendix 2: Computation of $G(y)$}

By the definition of $F$ in (4), the $j$-th component of the vector field $G$ defined in (10) can be written as

$$
\begin{aligned}
G_{j}(y) & =\left(C^{-1} f\left(C y+x_{+}^{*}\right)\right)_{j} \\
& =\sum_{k=1}^{4} C_{j k}^{-1}\left(\sum_{l=1}^{4} C_{k l} y_{l}+x_{+, 1}^{*}\right) F_{k} \\
& =\sum_{k, l=1}^{4} C_{j k}^{-1} C_{k l} y_{l} F_{k}+\sum_{k=1}^{4} C_{j k}^{-1} x_{+, 1}^{*} F_{k}
\end{aligned}
$$

where $F_{k}$ stands for $F_{k}\left(C y+x_{+}^{*}\right), \quad x_{+}^{*}=$ $\left(x_{+, 1}^{*}, x_{+, 1}^{*}, x_{+, 1}^{*}, x_{+, 1}^{*}\right)$, the notation $A_{j k}$ denotes the 
entry of a matrix $A$ located in the $j$ th row and $k$-th column, $C$ is defined in (9) and

$$
C^{-1}=\frac{1}{4}\left(\begin{array}{cccc}
2 & 0 & -2 & 0 \\
0 & 2 & 0 & -2 \\
1 & -1 & 1 & -1 \\
1 & 1 & 1 & 1
\end{array}\right)
$$

Next, we reduce the expression of the components of $F(C y+$ $\left.x_{+}^{*}\right)$ as:

$$
\begin{aligned}
F_{j}\left(C y+x_{+}^{*}\right)= & a(Q-1)+Q\left(C y+x_{+}^{*}\right)_{j-1} \\
& -\sum_{k=1}^{4}\left(C y+x_{+}^{*}\right)_{k}\left(C y+x_{+}^{*}\right)_{k-1} \\
= & a(Q-1)+Q(C y)_{j-1}+Q x_{+, 1}^{*} \\
& -\sum_{k=1}^{4}(C y)_{k}(C y)_{k-1} \\
& -\sum_{k=1}^{4} x_{+, 1}^{*}\left((C y)_{k}+(C y)_{k-1}\right)-4 x_{+, 1}^{*}{ }^{2} \\
= & Q(C y)_{j-1}-\sum_{k=1}^{4}(C y)_{k}(C y)_{k-1} \\
& -\sum_{k=1}^{4} x_{+, 1}^{*}\left((C y)_{k}+(C y)_{k-1}\right),
\end{aligned}
$$

subscripts $j$ and $k$ indicate components of vectors and in the last step we have used equation (6), and $(C y)_{0}:=(C y)_{4}$. Note that the last expression in the expansion of $F_{j}$ only contains one term that depends on $j, Q(C y)_{j-1}$, which can be computed directly from the definition of $C$ in (9). The other two terms are the same for every $j \in\{1,2,3,4\}$ and can be further simplified:

- The linear term $-\sum_{k=1}^{4} x_{+, 1}^{*}\left((C y)_{k}+(C y)_{k-1}\right)$, thanks to the cyclic structure of the variables, satisfies $\sum_{k=1}^{4}\left((C y)_{k}+(C y)_{k-1}\right)=2 \sum_{k=1}^{4}(C y)_{k}$. Moreover, since $\sum_{k=1}^{4} C_{k m}=0$ for $m=1,2,3$ and $\sum_{k=1}^{4} C_{k 4}=4$, then

$$
\sum_{k=1}^{4}(C y)_{k}=\sum_{k=1}^{4} \sum_{m=1}^{4} C_{k m} y_{m}=\sum_{m=1}^{4} y_{m} \sum_{k=1}^{4} C_{k m}=4 y_{4} .
$$

We conclude that

$$
-\sum_{k=1}^{4} x_{+, 1}^{*}\left((C y)_{k}+(C y)_{k-1}\right)=-8 x_{+, 1}^{*} y_{4} .
$$

- For the quadratic term $-\sum_{k=1}^{4}(C y)_{k}(C y)_{k-1}$, if we call $C_{k}$ the $k$-th row of matrix $C$, then it is easy to prove that

$$
\sum_{k=1}^{4}(C y)_{k}(C y)_{k-1}=y^{\top} M y
$$

where $M:=\sum_{k=1}^{4} C_{k}^{\top} C_{k-1}$, assuming that $C_{0}:=C_{4}$. A straightforward computation gives

$$
\begin{aligned}
M= & \left(\begin{array}{cccc}
0 & -2 & 0 & 0 \\
2 & 0 & 0 & 0 \\
0 & 0 & -4 & 0 \\
0 & 0 & 0 & 4
\end{array}\right), \text { and so } \\
& -\sum_{k=1}^{4}(C y)_{k}(C y)_{k-1}=4 y_{3}^{2}-4 y_{4}^{2} .
\end{aligned}
$$

Gathering the simplified expressions for the three terms from (23), we get:

$$
F_{j}\left(C y+x_{+}^{*}\right)=Q(C y)_{j-1}-8 x_{+, 1}^{*} y_{4}+4 y_{3}^{2}-4 y_{4}^{2} .
$$

We split this expression into $F_{j}\left(C y+x_{+}^{*}\right)=: F_{j}[1]+F[2]$, for each $j=1,2,3,4$, according to the degree (notice that the quadratic term does not depend on $j$ ), where

$$
\begin{aligned}
F_{j}[1] & =Q(C y)_{j-1}-8 x_{+, 1}^{*} y_{4}, \\
F[2] & =4 y_{3}^{2}-4 y_{4}^{2} .
\end{aligned}
$$

We plug the above expression for $F_{j}$ into the expression of $G_{j}$ obtained in (22):

$$
\begin{aligned}
G_{j}= & \sum_{k, l=1}^{4} C_{j k}^{-1} C_{k l} y_{l}\left(F_{k}[1]+F[2]\right) \\
& +\sum_{k=1}^{4} C_{j k}^{-1} x_{+, 1}^{*}\left(F_{k}[1]+F[2]\right) .
\end{aligned}
$$

The terms of order 1, 2 and 3 in $y$ are then:

$$
\begin{aligned}
G_{j}[1]= & \sum_{k=1}^{4} C_{j k}^{-1} x_{+, 1}^{*} F_{k}[1], \\
G_{j}[2]= & \sum_{k, l=1}^{4} C_{j k}^{-1} C_{k l} y_{l} F_{k}[1] \\
& +\sum_{k=1}^{4} C_{j k}^{-1} x_{+, 1}^{*} F[2], \\
G_{j}[3]= & \sum_{k, l=1}^{4} C_{j k}^{-1} C_{k l} y_{l} F[2],
\end{aligned}
$$

which, after substitution of $F_{k}[1]$ and $F[2]$, become:

$$
\begin{aligned}
G_{j}[1]= & \sum_{k=1}^{4} C_{j k}^{-1} x_{+, 1}^{*}\left(Q(C y)_{k-1}-8 x_{+, 1}^{*} y_{4}\right), \\
G_{j}[2]= & \sum_{k, l=1}^{4} C_{j k}^{-1} C_{k l} y_{l}\left(Q(C y)_{k-1}-8 x_{+, 1}^{*} y_{4}\right) \\
& +x_{+, 1}^{*}\left(4 y_{3}^{2}-4 y_{4}^{2}\right) \sum_{k=1}^{4} C_{j k}^{-1}, \\
G_{j}[3]= & \left(4 y_{3}^{2}-4 y_{4}^{2}\right) \sum_{k, l=1}^{4} C_{j k}^{-1} C_{k l} y_{l} .
\end{aligned}
$$

We develop each term separately, just substituting the entries 
of $C^{-1}$ and $C$, respectively, in the following two steps:

$$
\begin{aligned}
& G[1]= \\
& =x_{+, 1}^{*}\left(\begin{array}{c}
Q \frac{1}{2}\left((C y)_{4}-(C y)_{2}\right) \\
Q \frac{1}{2}\left((C y)_{1}-(C y)_{3}\right) \\
Q \frac{1}{4}\left((C y)_{4}-(C y)_{1}+(C y)_{2}-(C y)_{3}\right) \\
Q \frac{1}{4}\left((C y)_{4}+(C y)_{1}+(C y)_{2}+(C y)_{3}\right)-8 x_{+, 1}^{*} y_{4}
\end{array}\right) \\
& =Q x_{+, 1}^{*}\left(\begin{array}{c}
-y_{2} \\
y_{1} \\
-y_{3} \\
y_{4}
\end{array}\right)+\left(\begin{array}{c}
0 \\
0 \\
0 \\
-8 x_{+, 1}^{*}{ }^{2} y_{4}
\end{array}\right) .
\end{aligned}
$$

This gives the linear term provided in (11), just recalling the definitions of $\kappa=Q x_{+, 1}^{*}$ and $c=-\left(Q x_{+, 1}^{*}-8 x_{+, 1}^{*}{ }^{2}\right)=-Q x_{+, 1}^{*}+$ $2 Q x_{+, 1}^{*}+2 a(Q-1)=\kappa-2 a(1-Q)$.

The quadratic terms are given by the two terms of $G_{j}[2]$ in (24), for $j=1,2,3,4$. On one hand, one term only influences the fourth component:

$$
x_{+, 1}^{*}\left(4 y_{3}^{2}-4 y_{4}^{2}\right) \sum_{k=1}^{4} C_{j k}^{-1}=\left\{\begin{array}{l}
0 \text { if } j=1,2,3 \\
x_{+, 1}^{*}\left(4 y_{3}^{2}-4 y_{4}^{2}\right) \text { if } j=4 .
\end{array}\right.
$$

On the other hand, the term $\sum_{k, l=1}^{4} C_{j k}^{-1} C_{k l} y_{l}\left(Q(C y)_{k-1}-\right.$ $\left.8 x_{+, 1}^{*} y_{4}\right)$ requires more detailed computations. For $j=1$,

$$
\begin{aligned}
& \sum_{k, l=1}^{4} C_{1 k}^{-1} C_{k l} y_{l}\left(Q(C y)_{k-1}-8 x_{+, 1}^{*} y_{4}\right) \\
= & \sum_{k=1,3}(-1)^{\frac{k-1}{2}} \frac{1}{2} \sum_{l=1}^{4} C_{k l} y_{l}\left(Q(C y)_{k-1}-8 x_{+, 1}^{*} y_{4}\right) \\
= & \frac{1}{2}\left(y_{1}+y_{3}+y_{4}\right)\left(Q\left(-y_{2}-y_{3}+y_{4}\right)-8 x_{+, 1}^{*} y_{4}\right) \\
& -\frac{1}{2}\left(-y_{1}+y_{3}+y_{4}\right)\left(Q\left(y_{2}-y_{3}+y_{4}\right)-8 x_{+, 1}^{*} y_{4}\right) \\
= & Q\left(-y_{1} y_{3}+y_{1} y_{4}-y_{2} y_{3}-y_{2} y_{4}\right)-8 x_{+, 1}^{*} y_{1} y_{4},
\end{aligned}
$$

which coincides with the first component of $G[2]$ in (11). Similarly, for $j=2$ :

$$
\begin{aligned}
& \sum_{k, l=1}^{4} C_{2 k}^{-1} C_{k l} y_{l}\left(Q(C y)_{k-1}-8 x_{+, 1}^{*} y_{4}\right) \\
= & \sum_{k=2,4}(-1)^{\frac{k-2}{2}} \frac{1}{2} \sum_{l=1}^{4} C_{k l} y_{l}\left(Q(C y)_{k-1}-8 x_{+, 1}^{*} y_{4}\right) \\
= & \frac{1}{2}\left(y_{2}-y_{3}+y_{4}\right)\left(Q\left(y_{1}+y_{3}+y_{4}\right)-8 x_{+, 1}^{*} y_{4}\right) \\
& -\frac{1}{2}\left(-y_{2}-y_{3}+y_{4}\right)\left(Q\left(-y_{1}+y_{3}+y_{4}\right)-8 x_{+, 1}^{*} y_{4}\right) \\
= & Q\left(-y_{1} y_{3}+y_{1} y_{4}+y_{2} y_{3}+y_{2} y_{4}\right)-8 x_{+, 1}^{*} y_{2} y_{4},
\end{aligned}
$$

which coincides with the second component of $G[2]$ in (11). For $j=3$ :

$$
\begin{aligned}
& \sum_{k, l=1}^{4} C_{3 k}^{-1} C_{k l} y_{l}\left(Q(C y)_{k-1}-8 x_{+, 1}^{*} y_{4}\right) \\
= & \sum_{k=1}^{4}(-1)^{k-1} \frac{1}{4} \sum_{l=1}^{4} C_{k l} y_{l}\left(Q(C y)_{k-1}-8 x_{+, 1}^{*} y_{4}\right) \\
= & \frac{1}{4}\left(y_{1}+y_{3}+y_{4}\right)\left(Q\left(-y_{2}-y_{3}+y_{4}\right)-8 x_{+, 1}^{*} y_{4}\right) \\
& -\frac{1}{4}\left(y_{2}-y_{3}+y_{4}\right)\left(Q\left(y_{1}+y_{3}+y_{4}\right)-8 x_{+, 1}^{*} y_{4}\right) \\
& +\frac{1}{4}\left(-y_{1}+y_{3}+y_{4}\right)\left(Q\left(y_{2}-y_{3}+y_{4}\right)-8 x_{+, 1}^{*} y_{4}\right) \\
& -\frac{1}{4}\left(-y_{2}-y_{3}+y_{4}\right)\left(Q\left(-y_{1}+y_{3}+y_{4}\right)-8 x_{+, 1}^{*} y_{4}\right) \\
= & Q\left(-y_{1} y_{2}\right)-8 x_{+, 1}^{*} y_{3} y_{4},
\end{aligned}
$$

which coincides with the third component of $G[2]$ in (11). For $j=4$ :

$$
\begin{aligned}
& \sum_{k, l=1}^{4} C_{4 k}^{-1} C_{k l} y_{l}\left(Q(C y)_{k-1}-8 x_{+, 1}^{*} y_{4}\right) \\
= & \sum_{k=1}^{4} \frac{1}{4} \sum_{l=1}^{4} C_{k l} y_{l}\left(Q(C y)_{k-1}-8 x_{+, 1}^{*} y_{4}\right) \\
= & \frac{1}{4}\left(y_{1}+y_{3}+y_{4}\right)\left(Q\left(-y_{2}-y_{3}+y_{4}\right)-8 x_{+, 1}^{*} y_{4}\right) \\
& +\frac{1}{4}\left(y_{2}-y_{3}+y_{4}\right)\left(Q\left(y_{1}+y_{3}+y_{4}\right)-8 x_{+, 1}^{*} y_{4}\right) \\
& +\frac{1}{4}\left(-y_{1}+y_{3}+y_{4}\right)\left(Q\left(y_{2}-y_{3}+y_{4}\right)-8 x_{+, 1}^{*} y_{4}\right) \\
& +\frac{1}{4}\left(-y_{2}-y_{3}+y_{4}\right)\left(Q\left(-y_{1}+y_{3}+y_{4}\right)-8 x_{+, 1}^{*} y_{4}\right) \\
= & Q\left(-y_{3}^{2}+y_{4}^{2}\right)-8 x_{+, 1}^{*} y_{4}^{2},
\end{aligned}
$$

which, after adding the expression $x_{+, 1}^{*}\left(4 y_{3}^{2}-4 y_{4}^{2}\right)$ obtained above, coincides with the last component of $G[2]$ in (11).

Finally, similar computations lead to:

$$
\begin{aligned}
G_{1}[3] & =\left(4 y_{3}^{2}-4 y_{4}^{2}\right) \sum_{k=1,3}(-1)^{\frac{k-1}{2}} \frac{1}{2} \sum_{l=1}^{4} C_{k l} y_{l} \\
& =\left(4 y_{3}^{2}-4 y_{4}^{2}\right)\left(\frac{1}{2}\left(y_{1}+y_{3}+y_{4}\right)-\frac{1}{2}\left(-y_{1}+y_{3}+y_{4}\right)\right) \\
& =\left(4 y_{3}^{2}-4 y_{4}^{2}\right) y_{1} ; \\
G_{2}[3] & =\left(4 y_{3}^{2}-4 y_{4}^{2}\right) \sum_{k=2,4}(-1)^{\frac{k-2}{2}} \frac{1}{2} \sum_{l=1}^{4} C_{k l} y_{l} \\
& =\left(4 y_{3}^{2}-4 y_{4}^{2}\right)\left(\frac{1}{2}\left(y_{2}-y_{3}+y_{4}\right)-\frac{1}{2}\left(-y_{2}-y_{3}+y_{4}\right)\right) \\
& =\left(4 y_{3}^{2}-4 y_{4}^{2}\right) y_{2} ; \\
G_{3}[3] & =\left(4 y_{3}^{2}-4 y_{4}^{2}\right) \sum_{k=1}^{4}(-1)^{k-1} \frac{1}{4} \sum_{l=1}^{4} C_{k l} y_{l} \\
& =\left(4 y_{3}^{2}-4 y_{4}^{2}\right) y_{3} ; \\
G_{4}[3] & =\left(4 y_{3}^{2}-4 y_{4}^{2}\right) \sum_{k=1}^{4} \frac{1}{4} \sum_{l=1}^{4} C_{k l} y_{l} \\
& =\left(4 y_{3}^{2}-4 y_{4}^{2}\right) y_{4},
\end{aligned}
$$

as in (11) (notice that, in the last two steps, for the sake of the presentation, we do not display the full expansion in terms of the $C_{k l}$ elements). This completes the computation to obtain the expressions in (11) from (10). 